\title{
Correction to: Serum fatty acid-binding protein 4 (FABP4) as a novel biomarker to predict gestational diabetes mellitus
}

\author{
Hui Ning ${ }^{1,2} \cdot$ Hong Tao $^{2} \cdot$ Zhanping Weng $^{2} \cdot$ Xingbo Zhao $^{1}$
}

Published online: 18 July 2018

c) Springer-Verlag Italia S.r.l., part of Springer Nature 2018

\section{Correction to: Acta Diabetol (2016) 53:891-898 https://doi.org/10.1007/s00592-016-0867-8}

The title "Plasma fatty acid-binding protein 4 (FABP4) as a novel biomarker to predict gestational diabetes mellitus" should be replaced by "Serum fatty acid-binding protein 4 (FABP4) as a novel biomarker to predict gestational diabetes mellitus".

Conclusions of the Abstract should be replaced by the following sentence:

These results suggest that serum FABP4 may potentially serve as a novel biomarker for GDM.

The following sentence in the Introduction should be deleted:

"Although increased serum FABP4 levels were found in patients with GDM [1-3], it is unknown whether the level of serum FABP4 is a biomarker for prediction and risk of GDM"

The paragraph Biochemical analysis should be replaced by the following text:

$10 \mathrm{~mL}$ blood sample was collected from each patient into a tube after overnight fasting. Blood sample was immediately centrifuged to separate the serum, which was then stored at $-80{ }^{\circ} \mathrm{C}$ for a maximum of 12 months until further analysis. All serum samples were analysed in parallel to minimize variances.

The paragraph Statistical analysis should be replaced by the following text:

The data were first analyzed by Kolmogorov-Smirnov test to determine the distribution of data. Student $t$ test was used for normally distributed data, while Mann-Whitney $U$ test was used for non-normally distributed data.

In the paragraph "Subjects characteristics" (section Results) the following sentence "The LGA (large for gestational age) frequency was $8.33 \%$ in the GDM group and $4.44 \%$ in the control group. There were more infants with Apgar scores $<7$ at $5 \mathrm{~min}$ in the GDM group than in the control group (5.56 vs. $2.22 \%$ )" should be replaced by "The LGA (large for gestational age) frequency was $6.52 \%$ in the GDM group and $3.64 \%$ in the control group. There were more infants with Apgar scores $<7$ at $5 \mathrm{~min}$ in the GDM group than in the control group (4.35 vs. $1.82 \%)$ ".

Xingbo Zhao

xingboozhao@sina.com

1 Department of Obstetrics and Gynaecology, Shandong Provincial Hospital Affiliated to Shandong University, Jinan 250012, Shandong, China

2 Department of Obstetrics and Gynaecology, Qingdao Municipal Hospital, Qingdao 266000, Shandong, China 